Review Paper

\title{
Graphene Doping: A Review
}

\author{
Beidou Guo ${ }^{1,2}$, Liang Fang ${ }^{1, *}$, Baohong Zhang ${ }^{2}$, Jian Ru Gong ${ }^{2, *}$ \\ 1 Department of Applied Physics, Chongqing University, Chongqing 400044, China \\ 2 Laboratory for Nanodevices, National Center for Nanoscience and Technology, China, 11 \\ Zhongguancun Beiyitiao, Beijing 100190, P. R. China \\ * Author to whom correspondence should be addressed; E-Mails: gongjr@ nanoctr.cn, \\ fangliangcqu@yahoo.com.cn.
}

Received: 29 December 2010 / in revised form: 11 March 2011 / Accepted: 22 April 2011 / Published: 27 April 2011

\begin{abstract}
Graphene, a new material for the electron-device community, has many extraordinary properties. Especially, it provides a perfect platform to explore the unique electronic property in absolutely two-dimensions. However, most electronic applications are handicapped by the absence of a semiconducting gap in pristine graphene. To control the semiconducting properties of graphene, doping is regarded as one of the most feasible methods. Herein, a brief review is given on the recent research progress of graphene doping, which is roughly divided into three categories: First, the hetero atom doping, including arc discharge, chemical vapor deposition, electrothermal reaction and ion-irradiation approaches; Second, the chemical modification strategy; Third, the method of electrostatic field tuning. In addition, the various potential applications of the above doping methods are also introduced.
\end{abstract}

Keywords: graphene; electronic property; doping; molecular modification; electrostatic field. 


\section{Introduction}

Graphene, a single layer of graphite, has raised extensive interest in a wide scientific community for its extraordinary thermal, mechanical, electrical and other properties ${ }^{[1,2]}$. Among all properties, the unique electronic properties are assumed to be the most intriguing aspect of graphene, for example, outstanding ballistic transport properties and longest mean free path at room temperature ${ }^{[3]}$, distinctive integral and half-integral quantum hall effect ${ }^{[4,5]}$, the highest mobility ${ }^{[6]}$ to increase the speed of devices, and so on. The mobility of graphene is significantly higher than that of the widely-used semiconductor - $\mathrm{Si}$, of approximately $1400 \mathrm{~cm}^{2} \mathrm{~V}^{-1} \mathrm{~s}^{-1}$. Consequently, graphene has been considered as a candidate material for applications in post-silicon electronics.

However, most electronic applications are handicapped by the absence of a semiconducting gap in pristine graphene. For example, the devices made from the zero-bandgap graphene are difficult to switch off, losing the advantage of the low static power consumption of the complementary metal oxide semiconductor (CMOS) technology. Quantitatively, the $\mathrm{I}_{\mathrm{on}} / \mathrm{I}_{\text {off }}$ ratios for graphene-based fieldeffect transistors (GFETs) are less than 100, while any successor to the Si MOSFET should have excellent switching capabilities in the range $10^{4}-10^{7[7]}$. Therefore, opening a sizeable and well-tuned bandgap in graphene is a significant challenge for graphene-based electron-devices. The schematic band structures of pristine graphene with a linear energy-momentum dispersion relation $(E= \pm \hbar v|\mathbf{K}|$, where, $v$ is the Fermi velocity) near the Dirac point ${ }^{[8,9]}$ is shown in Figure 1a. Its valence and conduction bands are cone-shaped and meet at the $\mathbf{K}$ point of the Brillouin zone. Figure $1 \mathrm{~b}$ and 1c illustrate the schematic band structures of $p$ - and $n$-typed graphene with the bandgap and Fermi levels $\left(\mathrm{E}_{\mathrm{f}}\right)$ lying in the valence and conduction band, respectively.

Figure 1. Schematic band structures of graphene. (a) Band structure of pristine graphene with zero bandgap. $\mathrm{E}_{\mathrm{f}}$ is at the cross-over point. Band structures of (b) $p$-type and (c) $n$-type graphene with the bandgap. $\mathrm{E}_{\mathrm{f}}$ lies in valence and conduction band, respectively.
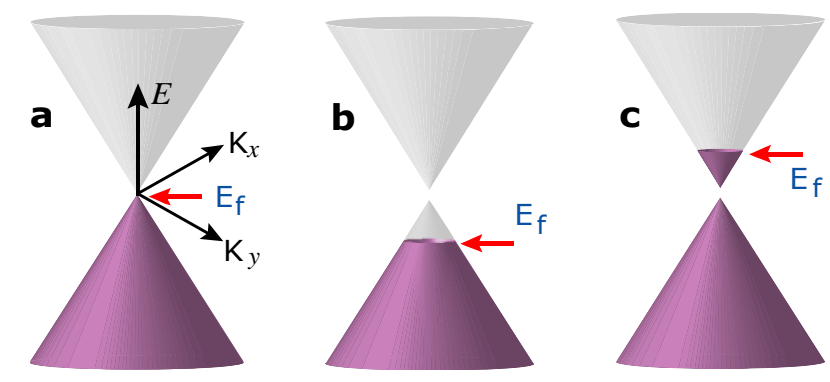

In this review, we will focus on a promising way of opening graphene bandgap — doping, examining the state-of-the-art doping methods, which are roughly classified into three categories: (1) Hetero atom doping; (2) Chemical modification; and (3) Electrostatic field tuning. The hetero atom doping and chemical modification methods can be used to open the bandgap and tune the Fermi level of graphene. While for the electrostatic field tuning method, the polarity and value of the gate voltage can change the Fermi level of graphene, but the bandgap cannot be opened. In addition, the various potential applications of the above-mentioned doping approaches are also briefly introduced. 


\section{Hetero atom doping}

Doping is the most feasible method to control the semiconducting properties in the conventional semiconductor community. The $\mathrm{B}$ and $\mathrm{N}$ atoms are the natural candidates for doping in graphene because of their similar atomic size as that of $\mathrm{C}$ and of their hole acceptor and electron donor characters for substitutional B- and N-doping, respectively.

The substitutional doping is also a useful method to open bandgap of graphene as proved by the theoretical work about B-, N- and Bi-doping in graphene ${ }^{[10-15]}$. Through first-principles density functional theory (DFT) and ab initio calculations, the effect of substitutional doping on the structure of graphene as well as on the electronic properties was studied ${ }^{[16]}$. The results reveal that the linearity in the dispersion of electronic bands within $1 \mathrm{eV}$ of the Fermi energy is almost unchanged with B- and $\mathrm{N}$-doping, indicating that the doped graphene exhibits the band structure with a linear dispersion relation similar to the band structure of pristine graphene. Nevertheless, bandgap is opened in graphene after substitutional doping with $\mathrm{B}$ and $\mathrm{N}$ atoms, and the Fermi level lies in valence and conduction band, respectively, showing ideal $p$ - and $n$-type semiconducting electronic properties as illustrated in Figure $1 \mathrm{~b}$ and $1 \mathrm{c}$ for potential applications of graphene in electronic devices.

Recently, several groups reported the B- and N-doping of graphene in the process of graphene synthesis. Arc discharge method, for example, was used to prepare B- and N-doped graphene by using high-current between graphite electrodes in the presence of $\mathrm{H}_{2}+\mathrm{B}_{2} \mathrm{H}_{6}$ and $\mathrm{H}_{2}+\mathrm{NH}_{3}$, respectively ${ }^{[16]}$. Liu group synthesized $\mathrm{N}$-doped graphene by chemical vapor deposition (CVD), using a 25-nm-thick $\mathrm{Cu}$ film on a Si substrate as the catalyst under $\mathrm{H}_{2}\left(20 \%\right.$ in $\mathrm{Ar}$ ) atmosphere and $\mathrm{CH}_{4}+\mathrm{NH}_{3}$ as the $\mathrm{C}$ and $\mathrm{N}$ source, respectively ${ }^{[17]}$. The X-ray photoelectron spectroscopy (XPS) data confirm the doping of graphene. The main peak at $284.8 \mathrm{eV}$ corresponds to the graphite-like $s p^{2} \mathrm{C}$, indicating most of the $\mathrm{C}$ atoms in the $\mathrm{N}$-doped graphene are arranged in a conjugated honeycomb lattice. They also fabricated FETs using doped graphene and measured the electronic properties of devices. Although the $\mathrm{N}$-doped graphene was successfully synthesized by CVD method, the mobility is less than $500 \mathrm{~cm}^{2} \mathrm{~V}^{-1} \mathrm{~s}^{-1}$, which is lower than that of the mechanical exfoliated graphene, $c a .5000 \mathrm{~cm}^{2} \mathrm{~V}^{-1} \mathrm{~s}^{-1[2]}$, and that of the suspended intrinsic graphene, as high as $200000 \mathrm{~cm}^{2} \mathrm{~V}^{-1} \mathrm{~s}^{-1[6]}$. Otherwise, most samples are few-layer graphene, and single-layer graphene can only be occasionally detected. Lately, Zhang et al. ${ }^{[18]}$ synthesized the $\mathrm{N}$-doped graphene using the embedded $\mathrm{C}$ and $\mathrm{N}$ by CVD method. The temperaturedependent electrical transport measurement showed that resistance of $\mathrm{N}$-doped graphene decreased by more than 80-fold when the temperature increased from liquid nitrogen temperature to $300 \mathrm{~K}$, indicating the semiconducting property of $\mathrm{N}$-doped graphene. The bandgap $\left(E_{g}\right)$ estimated based on the general relationship, $R(T) \propto \exp \left(E_{g} / 2 \kappa_{B} T\right)$, is about $0.16 \mathrm{eV}$, where all the symbols have their common meanings. Meyer and colleagues also investigated the electronic configuration of point defects and charge redistribution of $\mathrm{N}$-doped graphene prepared by CVD method using high-resolution transmission electron microscopy (HRTEM) ${ }^{[19]}$. It helps to understanding the effects of integrating hetero atoms into graphene on the local electronic properties ${ }^{[20]}$. After further improvement, CVD method will have great prospect for producing large-scale high-quality single-layer substitutional doped graphene.

Dai group at Stanford achieved N-doping in graphene nanoribbons (GNR) through electrothermal reaction with $\mathrm{NH}_{3}{ }^{[21,22]}$. The doping sites form $\mathrm{C}-\mathrm{N}$ bonds at the edges of the GNR. The N-doped GNR shows $n$-type electronic doping, which is consistent with the theoretical result. And the $\mathrm{I}_{\mathrm{on}} / \mathrm{I}_{\mathrm{off}}$ ratio of 
N-doped GNR-based FET is $c a .10^{5}$, larger than that of the pristine graphene-based $\mathrm{FET}^{[7]}$, but the mobility ranges from a few hundred to $\sim 1000 \mathrm{~cm}^{2} \mathrm{~V}^{-1} \mathrm{~s}^{-1}$, lower than that of pristine graphene ${ }^{[6]}$.

The intact pristine graphene has a perfect structure, and it is difficult to introduce foreign atoms in the whole plane. Gong group reported the $\mathrm{N}$-doping of graphene through $\mathrm{NH}_{3}$ annealing after $\mathrm{N}^{+}$-ion irradiation $^{[23]}$, and the stable and homogenous $\mathrm{N}$-doped graphene can be reproducibly obtained. According the procedure, the defects were firstly introduced in the plane of pristine graphene after irradiation. Raman spectroscopy was applied to monitor the amount of defects. After the samples were annealed in $\mathrm{NH}_{3}, \mathrm{~N}$ signal could be detected by Auger electron spectroscopy (AES), indicating that $\mathrm{N}$ atoms were introduced into $\mathrm{C}$ vacancies in the graphene. In the process of annealing, the defects of graphene were restored, getting the $\mathrm{N}$-doped graphene. The FETs were also fabricated using the doped graphene as channel material between source and drain electrodes as illustrated in Figure 2a (Left: the scheme of the graphene-based FET device; Right: a typical optical image of the device) to evaluate electrical properties. The $\mathrm{G}_{\mathrm{sd}}-\mathrm{V}_{\mathrm{g}}$ curve of the $\mathrm{N}$-doped GFET in Figure $2 \mathrm{~b}$ shows a clear $n$-type property with the minimum $\mathrm{G}_{\mathrm{sd}}$ locating at the negative gate voltage. The mobility of $\mathrm{N}$-doped graphene is about $6000 \mathrm{~cm}^{2} \mathrm{~V}^{-1} \mathrm{~s}^{-1}$, higher than the values reported by CVD method and comparable with that of pristine graphene ${ }^{[2]}$. Furthermore, the doping concentration can be controlled by tuning the specific conditions of irradiation and annealing, and it is also possible to replace the $\mathrm{N}$ atoms with other dopants. This approach is therefore an effective and universal method for graphene doping, and it is compatible with current integrated circuit CMOS. By choosing different dopants, the graphene-only $p$ - $n$ junctions can be fabricated using current semiconductor technology.

Figure 2. (a) The scheme of the graphene-based FET device (left) and a typical optical image of the device (right, the scale bar is 2 microns). (b) The $\mathrm{G}_{\mathrm{sd}}-\mathrm{V}_{\mathrm{g}}$ curve of the $\mathrm{N}$-doped graphene device.

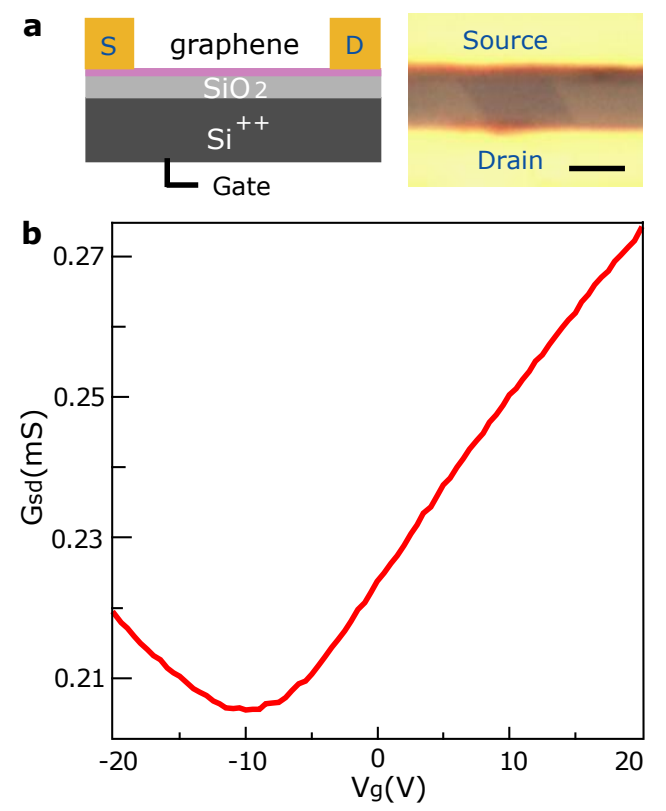




\section{Chemical modification}

As a two-dimentional (2D) material with only one atom layer thick, graphene has an absolute maximum of the surface area to volume ratio. Otherwise, considering its unique electronic ambipolarity for FET, it would be sensitive to molecular modification, and the molecule of either hole acceptor or electron donor would lead to $p$ - or $n$-type characters, respectively. Therefore, graphene can obtain fine control of electronic properties by the chemical modification ${ }^{[24]}$.

Some researchers have studied the properties of graphene modified by $\mathrm{NO}_{2}$ and $\mathrm{NH}_{3}$ and confirmed the opposite sign of carriers generated by $\mathrm{NO}_{2}$ and $\mathrm{NH}_{3}$, with a hole acceptor inducing conduction by holes and electron donor inducing conduction by electrons, respectively. Moreover, the molecular modification induced impurities and scatters, but the electrical properties of the GFET, such as carrier mobilities, did not degrade significantly. And this property can be used for high-sensitivity single molecule detection ${ }^{[24]}$.

Spin-polarized DFT calculations show that adsorption of water molecules on graphene plays the role of defects which facilitates the tunability of the bandgap and results in opening a large bandgap of $\sim 2 \mathrm{eV}^{[25]}$. Researchers recently exposed the graphene film to humility and created a bandgap in graphene successfully ${ }^{[26]}$. The bandgap increases with the increasing of humidity and saturates at $0.206 \mathrm{eV}$ at the humidity level of $c a .0 .312 \mathrm{~kg} \mathrm{~kg}^{-1}$, consistent with the theoretical result ${ }^{[25]}$. This method is inexpensive, nontoxic, and much easier to control for a chip application. However, the performance of the device might degrade after operation in a humid atmosphere for a long time.

Research results also show a hint that organic molecules, for instance, nonaromatic or aromatic molecules, are good choices to control graphene doping ${ }^{[27-31]}$. It provides a promising approach to control the carrier type and concentration using molecules adsorbed on graphene. Coletti et al. ${ }^{[32]}$ demonstrated that tetrafluorotetracyanoquinodimethane (F4-TCNQ), a hole acceptor, can be used to tune the Fermi level of graphene by noncovalent functionalization. What's more, aromatic molecules are good candidates for the modulation of the electronic structures via strong $\pi-\pi$ interaction between their aromatic rings and graphene ${ }^{[33]}$. Electrical experiments on the GFETs suggest that the aromatic molecules with electron-donor groups (e.g., 9,10-Dimethylanthracene $\left(\mathrm{An}-\mathrm{CH}_{3}\right)$, 1,5Naphthalenediamine $\left(\mathrm{Na}-\mathrm{NH}_{2}\right)$ ) cause $n$-doping, while those with hole acceptor groups impose $p$ doping (e.g., 9,10-Dibromo-anthracene (An-Br), tetrasodium 1,3,6,8-pyrenetetrasulfonic acid (TPA) ) on graphene. Rich knowledge from the rapidly developing organic electronics will contribute to the further development of various molecule-modified $p$ - or $n$-typed graphene, and the research of using molecular modification method to fabricate junction was reported by Farmer et al. through exposing the conductive channel of GFET partially to the diazonium salt ${ }^{[34]}$.

Electrochemical method was also used in the area of doping of graphene ${ }^{[35]}$. Chen et al. ${ }^{[36]}$ have studied the charge transport of single layer graphene transistors via an electrochemical tuning approach in ionic liquids and aqueous solutions. The changes of carrier type and concention induced by electrochemical doping can be detected by Raman spectroscopy directly ${ }^{[37,38]}$. 


\section{Electrostatic field tuning}

Besides the two methods mentioned above, electrostatic field tuning can also be used to control electrical and magnetic properties of graphene by controlling the reversible changes of carrier concentration and the Fermi level without increasing the level of disorders and defects in graphene ${ }^{[39,40]}$. And the application of the FET principle to graphene to achieve electrostatic doping is a relatively important research area.

Top- and back-gate FETs are the prevalent manners for electrostatic field tuning ${ }^{[8]}$. Using this method, the Fermi level of pristine graphene can be finely tuned from conduction band to valence band, following the change of the gate voltage from negative to positive, corresponding to the $p$ - and $n$-typed graphene. In this way, the electrostatic potential barrier can be made using either top- or backgate voltage. As illustrated in Figure 3, graphene is placed over $\mathrm{SiO}_{2} / \mathrm{p}^{++} \mathrm{Si}$ substrate, and the topelectrode (top-gate) was used to produce the $n$-doped area, while the back-gate for the $p$-doped region. The Klein tunneling, which means that carriers can tunnel the barrier induced by the gate voltage ${ }^{[41]}$, in graphene heterojunctions has been reported in $p-n$ junction, $p-n-p$ or $n-p-n$ junction by several groups ${ }^{[42-45]}$, different from the conventional $p-n$ junction with the unilateral conductive property ${ }^{[46-48]}$. Considering the merit of Klein tunneling, graphene can be used to make atomic clock, scanning tunneling microscope and tunneling transistor, and so on. Cheianov et al. ${ }^{[4]}$ have successfully predicted the focusing of electron flow by the single $p$ - $n$ junction in graphene, and the result is promising for making a Veselago lens which uses electrons for the 3D imaging. Recently, the electronic analogues of optical devices with both positive and negative indices of refraction were reported by Williams et al. by tuning the $p$ - and $n$-typed carrier density in graphene through the gate voltage ${ }^{[50]}$.The research may lead to a new functionality in microelectronics. Liu et al. also observed coulomb blockade in GRN-FET with a top-gate ${ }^{[51]}$, finding that the electrons could be confined by the barriers induced by $p-n$ junctions created via electrostatic gates.

Although abundant excellent results have been reported, it should be noted that the electrostatic field tuning method cannot open bandgap in single layer graphene so far ${ }^{[52-54]}$. Therefore, this method has limited applications and much improvements are needed.

A few other ways to modify the band structure of graphene for possible bandgap opening were also reported recently, such as cutting graphene into nanoribbon ${ }^{[55,56]}$, using contacts between graphene and metals ${ }^{[57,58]}$, applying strain to graphene ${ }^{[59,60]}$ and biasing bilayer graphene ${ }^{[61-63]}$. Due to the limit of the doping topic in this review, these methods will not be further introduced.

In summary, the recent research advances on graphene doping is briefly reviewed, mainly including hetero atom doping, chemical modification and the electrostatic field tuning three categories. These are promising ways to open a sizeable and well-tuned bandgap in graphene, making graphene an excellent candidate for the applications in the circuits beyond the conventional CMOS technology and many other potential applications. 
Figure 3. Graphene $p$ - $n$ junction. (a) The scheme of the graphene-based dual-gate FET device. (b) The energy diagram shows a potential barrier with height $V$ and the position of the Fermi level with respect to the touching point of the valence and the conduction bands.
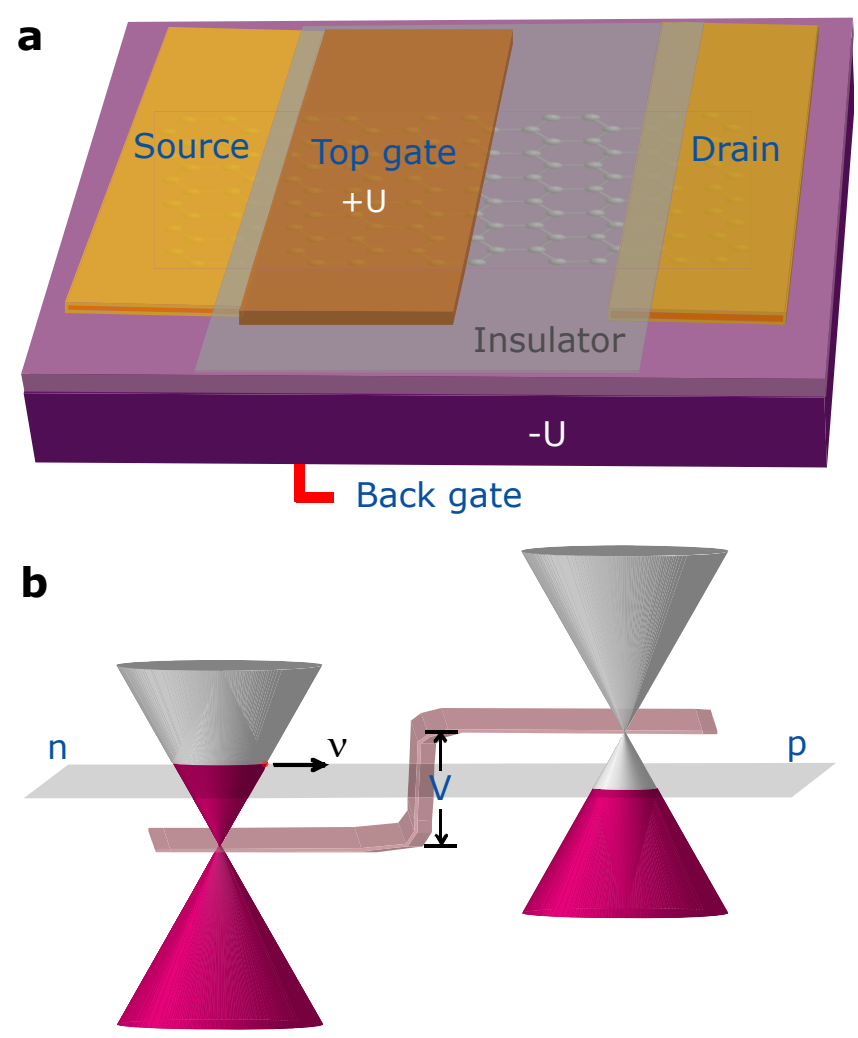

\section{Acknowledgement}

Prof. Jian Ru Gong sincerely thanks the financial support from National Basic Research Program of China (973 Program, No. 2011CB933401), Special Presidential Foundation of Chinese Academy of Sciences and National Natural Science Foundation of China (No. 21005023).

\section{References}

1. Geim, A. K. Science 2009, 324 (5934), 1530-1534.

2. Geim, A. K.; Novoselov, K. S. Nat. Mater. 2007, 6 (3), 183-191.

3. Gunlycke, D.; Lawler, H. M.; White, C. T. Phys. Rev. B 2007, 75 (8), 085418.

4. Novoselov, K. S.; Jiang, Z.; Zhang, Y.; Morozov, S. V.; Stormer, H. L.; Zeitler, U.; Maan, J. C.; Boebinger, G. S.; Kim, P.; Geim, A. K. Science 2007, 315 (5817), 1379.

5. Zhang, Y.; Tan, Y.-W.; Stormer, H. L.; Kim, P. Nature 2005, 438 (7065), 201-204.

6. Du, X.; Skachko, I.; Barker, A.; Andrei, E. Y. Nat. Nanotech. 2008, 3 (8), 491-495.

7. Schwierz, F. Nat. Nanotech. 2010, 5 (7), 487-496.

8. Castro Neto, A. H.; Guinea, F.; Peres, N. M. R.; Novoselov, K. S.; Geim, A. K. Rev. Mod. Phys. 2009, 81 (1), 109. 
9. Novoselov, K. S.; Morozov, S. V.; Mohinddin, T. M. G.; Ponomarenko, L. A.; Elias, D. C.; Yang, R.; Barbolina, I. I.; Blake, P.; Booth, T. J.; Jiang, D.; Giesbers, J.; Hill, E. W.; Geim, A. K. Phys. Status Solidi B 2007, 244 (11), 4106-4111.

10. Ci, L.; Song, L.; Jin, C.; Jariwala, D.; Wu, D.; Li, Y.; Srivastava, A.; Wang, Z. F.; Storr, K.; Balicas, L.; Liu, F.; Ajayan, P. M. Nat. Mater. 2010, 9 (5), 430-435.

11. Kotakoski, J.; Krasheninnikov, A. V.; Ma, Y.; Foster, A. S.; Nordlund, K.; Nieminen, R. M. Phys. Rev. B 2005, 71 (20), 205408.

12. Martins, T. B.; Miwa, R. H.; da Silva, A. J. R.; Fazzio, A. Phys. Rev. Lett. 2007, 98 (19), 196803.

13. Denis, P. A. Chem. Phys. Lett. 2010, 492 (4-6), 251-257.

14. Akturk, O. U.; Tomak, M. Appl. Phys.Lett. 2010, 96 (8), 081914-3

15. Yu, S.; Zheng, W.; Wang, C.; Jiang, Q. Acs Nano 2010, 4 (12), 7619-7629.

16. Panchokarla, L. S.; Subrahmanyam, K. S.; Saha, S. K.; Govindaraj, A.; Krishnamurthy, H. R.; Waghmare, U. V.; Rao, C. N. R. Adv. Mater. 2009, 21 (46), 4726-4730.

17. Wei, D. C.; Liu, Y. Q.; Wang, Y.; Zhang, H. L.; Huang, L. P.; Yu, G. Nano Lett. 2009, 9 (5), 1752-1758.

18. Zhang, C.; Fu, L.; Liu, N.; Liu, M.; Wang, Y.; Liu, Z. Adv. Mater. 2011, 23 (8), 1020-1024.

19. Meyer, J. C.; Kurasch, S.; Park, H. J.; Skakalova, V.; Künzel, D.; Groß, A.; Chuvilin, A.; Algara-Siller, G.; Roth, S.; Iwasaki, T.; Starke, U.; Smet, J. H.; Kaiser, U. Nat. Mater. 2011, 10 (3), 209-215.

20. Urban, K. W. Nat. Mater. 2011, 10 (3), 165-166.

21. Wang, X.; Li, X.; Zhang, L.; Yoon, Y.; Weber, P. K.; Wang, H.; Guo, J.; Dai, H. Science 2009, 324 (5928), 768-771.

22. Li, X. L.; Wang, H. L.; Robinson, J. T.; Sanchez, H.; Diankov, G.; Dai, H. J. J. Am. Chem. Soc. 2009, 131 (43), 15939-15944.

23. Guo, B.; Liu, Q.; Chen, E.; Zhu, H.; Fang, L.; Gong, J. R. Nano Lett. 2010, 10 (12), 4975-4980.

24. Schedin, F.; Geim, A. K.; Morozov, S. V.; Hill, E. W.; Blake, P.; Katsnelson, M. I.; Novoselov, K. S. Nat. Mater. 2007, 6 (9), 652-655.

25. Berashevich, J.; Chakraborty, T. Phys. Rev. B 2009, 80 (3), 033404.

26. Yavari, F.; Kritzinger, C.; Gaire, C.; Song, L.; Gulapalli, H.; Borca-Tasciuc, T.; Ajayan, P. M.; Koratkar, N. Small 2010, 6 (22), 2535-2538.

27. Pinto, H.; Jones, R.; Goss, P. J.; Briddon, R. P. J. Phys.: Condens. Matter 2009, 21 (40), 402001.

28. Hsieh, D.; Xia, Y.; Qian, D.; Wray, L.; Dil, J. H.; Meier, F.; Osterwalder, J.; Patthey, L.; Checkelsky, J. G.; Ong, N. P.; Fedorov, A. V.; Lin, H.; Bansil, A.; Grauer, D.; Hor, Y. S.; Cava, R. J.; Hasan, M. Z. Nature 2009, 460 (7259), 1101-1105.

29. Chen, W.; Qi, D.; Gao, X.; Wee, A. T. S. Prog. Surf. Sci. 84 (9-10), 279-321.

30. Oostinga, J. B.; Heersche, H. B.; Liu, X.; Morpurgo, A. F.; Vandersypen, L. M. K. Nat. Mater. 2008, 7 (2), 151-157.

31. Farmer, D. B.; Golizadeh-Mojarad, R.; Perebeinos, V.; Lin, Y.-M.; Tulevski, G. S.; Tsang, J. C.; Avouris, P. Nano Lett. 2008, 9 (1), 388-392.

32. Coletti, C.; Riedl, C.; Lee, D. S.; Krauss, B.; Patthey, L.; von Klitzing, K.; Smet, J. H.; Starke, U. Phys. Rev. B 2010, 81 (23), 235401. 
33. Dong, X.; Fu, D.; Fang, W.; Shi, Y.; Chen, P.; Li, L.-J. Small 2009, 5 (12), 1422-1426.

34. Farmer, D. B.; Lin, Y.-M.; Afzali-Ardakani, A.; Avouris, P. Appl. Phys. Lett. 2009, 94 (21), 213106-3.

35. Kalbac, M.; Reina-Cecco, A.; Farhat, H.; Kong, J.; Kavan, L.; Dresselhaus, M. S. Acs Nano 2010, 4 (10), 6055-6063.

36. Chen, F.; Qing, Q.; Xia, J.; Li, J.; Tao, N. J. Am. Chem. Soc. 2009, 131 (29), 9908-9909.

37. Das, A; Pisana, S; Chakraborty, B; Piscanec, S; Saha, S. K.; Waghmare, U. V.; Novoselov, K. S.; Krishnamurthy, H. R.; Geim, A. K.; Ferrari, A. C.; Sood, A. K. Nat. Nanotech. 2008, 3 (4), 210-215.

38. Panchakarla, L. S.; Govindaraj, A.; Rao, C. N. R. Inorg. Chim. Acta 2010, 363 (15), 41634174.

39. Ahn, C. H.; Bhattacharya, A.; Di Ventra, M.; Eckstein, J. N.; Frisbie, C. D.; Gershenson, M. E.; Goldman, A. M.; Inoue, I. H.; Mannhart, J.; Millis, A. J.; Morpurgo, A. F.; Natelson, D.; Triscone, J.-M. Rev. Mod. Phys. 2006, 78 (4), 1185.

40. Casiraghi, C. Phys. Rev. B 2009, 80 (23), 233407.

41. Dombey, N.; Calogeracos, A. Phys. Rep. 1999, 315 (1-3), 41-58.

42. Stander, N.; Huard, B.; Goldhaber-Gordon, D. Phys. Rev. Lett. 2009, 102 (2), 026807.

43. Young, A. F.; Kim, P. Nat. Phys. 2009, 5 (3), 222-226.

44. Bahat-Treidel, O.; Peleg, O.; Grobman, M.; Shapira, N.; Segev, M.; Pereg-Barnea, T. Phys. Rev. Lett. 2010, 104 (6), 063901.

45. Huard, B.; Sulpizio, J. A.; Stander, N.; Todd, K.; Yang, B.; Goldhaber-Gordon, D. Phys. Rev. Lett. 2007, 98 (23), 236803.

46. Chiu, H.-Y.; Perebeinos, V.; Lin, Y.-M.; Avouris, P. Nano Lett. 2010, 10 (11), 4634-4639.

47. Peters, E. C.; Lee, E. J. H.; Burghard, M.; Kern, K. Appl. Phys. Lett.2010, 97 (19), 193102-3.

48. Williams, J. R.; DiCarlo, L.; Marcus, C. M. Science 2007, 317 (5838), 638-641.

49. Cheianov, V. V.; Fal'ko, V.; Altshuler, B. L. Science 2007, 315 (5816), 1252-1255.

50. Williams, J. R.; Low, T.; Lundstrom, M. S.; Marcus, C. M. Nat. Nanotech. 2011, advance online publication. DOI: 10.1038/NNANO.2011.3

51. Liu, X.; Oostinga, J. B.; Morpurgo, A. F.; Vandersypen, L. M. K. Phys. Rev. B 2009, 80 (12), 121407.

52. Novoselov, K. S.; Geim, A. K.; Morozov, S. V.; Jiang, D.; Zhang, Y.; Dubonos, S. V.; Grigorieva, I. V.; Firsov, A. A. Science 2004, 306 (5696), 666-669.

53. Pisana, S.; Lazzeri, M.; Casiraghi, C.; Novoselov, K. S.; Geim, A. K.; Ferrari, A. C.; Mauri, F. Nat. Mater. 2007, 6 (3), 198-201.

54. Das, A; Pisana, S; Chakraborty, B; Piscanec, S; Saha, S. K.; Waghmare, U. V.; Novoselov, K. S.; Krishnamurthy, H. R.; Geim, A. K.; Ferrari, A. C.; Sood, A. K. Nat. Nanotech. 2008, 3 (4), 210-215.

55. Han, M. Y.; Ouml; zyilmaz, B.; Zhang, Y.; Kim, P. Phys. Rev. Lett. 2007, 98 (20), 206805.

56. Li, X.; Wang, X.; Zhang, L.; Lee, S.; Dai, H. Science 2008, 319 (5867), 1229-1232.

57. Giovannetti, G.; Khomyakov, P. A.; Brocks, G.; Karpan, V. M.; van den Brink, J.; Kelly, P. J. Phys. Rev. Lett. 2008, 101 (2), 026803.

58. Moktadir, Z.; Boden, S. A.; Ghiass, A.; Rutt, H.; Mizuta, H. Electron. Lett. 2011, 47 (3), 199200. 
59. Pereira, V. M.; Castro Neto, A. H.; Peres, N. M. R. Phys. Rev. B 2009, 80 (4), 045401.

60. Ni, Z. H.; Yu, T.; Lu, Y. H.; Wang, Y. Y.; Feng, Y. P.; Shen, Z. X. Acs Nano 2008, 2 (11), 2301-2305.

61. Castro, E. V.; Novoselov, K. S.; Morozov, S. V.; Peres, N. M. R.; dos Santos, J. M. B. L.; Nilsson, J.; Guinea, F.; Geim, A. K.; Neto, A. H. C. Phys. Rev. Lett. 2007, 99 (21), 216802.

62. Zhang, Y.; Tang, T.-T.; Girit, C.; Hao, Z.; Martin, M. C.; Zettl, A.; Crommie, M. F.; Shen, Y. R.; Wang, F. Nature 2009, 459 (7248), 820-823.

63. Ohta, T.; Bostwick, A.; Seyller, T.; Horn, K.; Rotenberg, E. Science 2006, 313 (5789), 951954.

(C) 2011 by the authors; licensee Insciences Journal.

Open Access article under the terms and conditions of Creative Commons Attribution Non-Commercial License 3.0 Unported. 